\title{
Discovering Cultural Differences to Inform Cultural Sensitive Nursing Practice
}

\author{
JieLi Li*1, Rasika Jayasekara ${ }^{2}$ and Ying Zhang ${ }^{3}$ \\ ${ }^{1}$ Bachelor degree of Medicine, Yunnan University of Traditional Chinese Medicine, china \\ ${ }^{2}$ Senior Lecturer in Nursing \& Midwifery, Australia \\ ${ }^{3}$ The First Affiliated Hospital of Yunnan University of Traditional Chinese Medicine, China
}

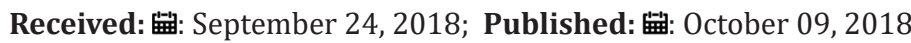

*Corresponding author: Jie Li Li, Bachelor degree of Medicine, Yunnan University of Traditional Chinese Medicine, Master of Nursing Practice Candidate, Monash University, Australia

\section{Introduction}

It is widely accepted that each cultural group possess their own values, beliefs, and perspectives to health. In order to provide culturally safe and respectful practice, Registered Nurses in Australia, are required to identify and explore their own culture, values, attitudes, assumptions and beliefs which influence their interactions with people and families, the community and colleagues (Nursing \& Midwifery of Australia (NMBA) [1]. In this paper, the discussion focuses on diverse health beliefs, health literacy, healthcare relationships and spiritual considerations between Chinese culture (mainland) and Australian mainstream culture. It will further illustrate the issues of culture clash between these two cultures and provide brief nursing strategies to reduce the cultural gaps. Despite various geographic features and socioeconomic backgrounds, people in mainland China share, to varying degrees, a heritage of Traditional Chinese Medicine (TCM) values handed down through their generations Chung et al. [2].

A most recent systematic review conducted by Chung et al. [2] indicates that TCM rhetoric is omnipresent in Chinese communities' everyday life. Most Chinese believe that the existence of good health is a result of the balance of Yin and Yang, and the harmonious flow of Qi and Blood in their bodies Chung et al. [2]. Illness occurs when your body has a violation against nature Chung et al. [2]. For them, Health promotion is achieved by adopting a balanced diet, utilizing natural substances and conducting traditional Chinese exercises Chung et al. [2]. This can be seen from examples in China such as tea therapy for PM 2.5 air pollution Zhang, Jiang \& Zhang [3] and ginger soup for common cold Chang, Wang, et al. [4]. In terms of hospital practice in China, modern medicine dominates almost every hospital across mainland China (Joe, 2012). TCM plays a complementary role when it comes to chronic diseases, rehabilitation, and home recovery (Joe, 2012). It is very common to have patients in mainland China received a western diagnosis, booked with laboratory and radiographic works, followed by a prescription of an antibiotic but separately being treated with acupuncture (Joe, 2012).
In contrast, due to historical reasons, Australian mainstream culture which evolved in western industrialized societies embraced a scientific approach towards people' health Willis [5]. People generally consider disease as a result of natural scientific phenomena caused by the microorganism such as bacteria or virus Willis [5]. Unlike in China, antibiotics are easily accessible and largely used by patients who have no medical background (Hao, Zhao, Qiu, Wang, \& Song [6] people in Australia purchase over-the-counter (OTC) drugs only for limited medical conditions Capon, Smith, \& Gillespie [7]. Consumers generally have an understanding that medications in Australia are strictly regulated by the government Capon et al. [7]. Regarding medical practice, treatment in Australian hospitals relies heavily upon industrially produced medications, complex surgeries, and formal scientific process Hodge [8]. That is to say, Chinese utilize mostly Western Medicine but also refers to TCM in everyday life while mainstream Australian mainly depend on Western Medicine. Health literacy indicates the degree to which individuals' capacity to obtain basic health information, to make appropriate health-related decisions and to follow the instructions for medical treatment. It plays a major role in people's health as well as patients' compliance in the hospital Wu et al. [8,9].

An alarming fact was that the national prevalence of low health literacy in China was documented as $93.52 \%$ Wu et al. [8,9]. While geographic disparity exists: developed city Jiangsu has $47.5 \%$ of low health literacy Wang et al. [10]; and capital city Beijing has $59 \%$ of communicable diseases health literacy Zhang et al. [11]. In a remote area of China, a hypertension knowledge survey revealed a score of 26/100 for hypertensive and 20/100 for non-hypertensive respondents Li et al. [12]. Hence, health literacy is a prominent issue for people in China especially those who live in a rural area of mainland China. In comparison, the situation in Australia is much more encouraging. It is well known that Australian enjoy one of the longest life expectancies in the world, and the better health literacy in Australia is a major contributing factor to the more significant health outcome Hall. The most recent data from the Australian Bureau of Statistics (ABS) confirms that. 
In 2006, $41 \%$ of Australian aged 15-74 were assessed as having adequate or more than adequate health literacy skills (ABS, 2008b, as cited in AIHW, 2018). Half (50\%) of the residents aged 30-39 had health literacy skills were adequate or even better (ABS, 2008b, as cited in AIHW, 2018). People aged 60-74 still maintained a percentage of $22 \%$ having adequate health literacy (ABS, 2008b, as cited in AIHW, 2018). To sum up, limited health literacy hinders a patient's motivation to self-care, intention to obtain professional advice, and wil Zhang gness to take early interventions. Therefore, it needs to be addressed promptly so as to promote better health for the public. China is widely acknowledged in the world that has a traditional culture of respecting the elderly people owing to the influence of Confucianism Gao, Wei, Shen, Tang, \& Yang et al. [13,14].

The Chinese government even has a range of laws such as Constitutional Law, Law on Protection of The Rights and Interests of The Elderly, Marriage Law and Criminal Law to enforce the legal obligation for children to support their parents financially and emotionally Yu [15]. The distinctive differences of elderly care between China and Australia are not the level of respect people pay to their older generation but their ways of caring them Gao Willis et al. $[5,14]$. Older couples live separately with their grown-up children and rely on their own pension or government subsidy is quite understandable in Australia Willis [5]. While in such a county like China that filial piety is rigidly expected, leaving your parents to live alone is regarded as absolutely immoral Gao et al. [14]. Social media even created a phrase for this "special phenomenon" as "empty nest", for those elderly being left alone are named "empty nesters" Gao et al. [14] Relocating older parents to an aged care facility will bring stigma to the whole family because the older parents will fell "being abandoned by their next generation" and the children will be criticized for being "unwilling or incapable of looking after their loved parents" Gao et al. [14].

Whereas residential nursing homes are generally accepted in Australia giving that different generations conceive varied lifestyles and require their own privacy Willis [5]. To conclude, these two cultures embrace a different concept of caring for their older parents. Moving on to the comparison of healthcare relationships between mainland China and Australia, there are substantial differences. Interestingly, with the economic development, globalization and open reform of China, there is a tendency of China to follow Australian healthcare relationships' trend. The consensus is that before China's economic reform and opening up in the 1980s, maintaining harmony is the most vital part of Chinese value Zhao, Zhang, Bai, \& Wang [16]. In the healthcare setting, mainly, conflict and direct confrontation were not socially accepted Smith, Fisher, \& Mercer [17]. Physicians and nurses were in a paternalistic position in most circumstances owning absolute authority and decisionmaking rights Smith et al. [17].

Disagreement with the recommended treatment was avoided Smith et al. [17]. However, over the past 10 years, the doctors /nurses -patient relationships in China have been changing dramatically due to factors such as dissatisfactory healthcare system, false media report and public low health literacy Jing et al.
[18]. It is a misfortune that an increasing number of Chinese doctors and nurses are becoming the victims of violent attacks and murders Jing, Zhao, et al. [5,7]. Take 2010 for example, there were more than "17,000 incidents" happened to health workers including a doctor being killed on 23rd March in Harbin, a specialist being stabbed on 13th April in Beijing, and a nurse being brutally attacked on 12th May in Nanjing (The Economist [19]. To some degree, the increase in violent assaults in healthcare workplace agrees with the current situation in Australia although the reasons for the tension differ. A retrospective review in New South Wales recorded 208 incidents of healthcare workplace violence in 2012 Cashmore, Indig, Hampton, Hegney, \& Jalaludin [20]. The Australian Nursing \& Midwifery Federation (ANMF) [1] undertook a study into occupational health and safety in the workplace in 2015 revealed that $70 \%$ of nurses, midwives, and carers surveyed had experienced aggression and violence in their past year at work. "While it was not just the frequency of incidents that was alarming but the severity of attacks is also increasing." Emphasized by the secretary Lisa Fitzpatrick of ANMF (Vic Branch) ANMF [3].

As we can see from the above data, the unfortunate reality is coincidently similar in both countries. Nevertheless, the degree of mistrust of patients towards the current medical system in China is far worse than which of Australia Yan [12]. Pleasantly, some promising changes have been happening in China because of the proliferation of "Zero Tolerance of Violence" policy and also the severe penalties applied to the attackers CDRF, 2018 [21]. Public social media also take actions to wisely direct agitated patients and inform them about how innocent healthcare professionals become the scapegoat of the poor healthcare system CDRF, 2018 [21]. Patients are becoming more and more rational in terms of seeking justice CDRF, 2018 [21]. Voilence towards health workers become less likely their top listed choices CDRF, 2018 [21]. Patients nowadays follow the westernized practice to seek third party opinions or legal protection when they feel they have not been appropriately treated (Lin, 2015). On the other hand, the authoritarian relationship is declining, especially in metropolitan cities in China CDRF, 2018 [21]. Instead of passively following doctors or nurses' orders, Chinese patients are becoming more and more involved in their treatment process (Lin, 2015). Just like medical practice in Australia: informed consent is to be obtained prior to significant invasive procedures (Lin, 2015); open disclosure is also encouraged (Lin, 18,2015); patients' rights are respected CDRF, 2018 [21].

All in all, there is still a long journey for China and Australia to continue so as to achieve a harmonious healthcare relationship. Cultural differences also influence people's way of understanding, managing and coping with the course of illnesses. A semi-structured interview of two cohort groups of Chinese Australian revealed several cultural clashes: seeking counselling or physiological help is considered humiliated for Chinese Australian because of cultural stigma of mental illness Ku, Maude, \& Welch [22]. Hiding their status of unemployment or not finding a spouse is a way of "saving face" $\mathrm{Ku}$ et al. [22]. Therefore, some anti-stigma nursing strategies such as community mental health education and cultural interventions 
can be implemented to improve their community attitudes. Again, in mental health research area, a Chinese woman has been observed to weep profusely when talking to anyone in a position of authority Tan, 2011. They want to find redress through weeping, and this behavior might continue for years even after the issue has been settled Tan, 2011. For this reason, the most appropriate nursing strategies are listening to them non-judgmentally, communicating to them therapeutically, and comforting them genially.

Apart from the above cultural conflicts, some traditional Chinese practices might seem unusual or even unhealthy to Australians. "Sitting-the-month", also named "zuoyuezi" in China, applies to the month after giving birth, when the new mum is expected to confine indoors and obey traditional rules to gain a good recovery Chen [22]. It is understood by the Chinese people that women lose a huge amount of blood during childbirth which leads to a cold and weak body Chen [22]. Hence, avoiding exposure to cold water, fresh fruits, ice, Salad is necessary Liu, Petrini, \& Maloni [13,24]. Nurses and midwives in Australia might find Chinese new mums not just refuse the cold sandwich or Salad provided by the hospital but also are reluctant to mobilize outside of the bed Liu et al. [13].

It is argued that although the rationale behind "sitting-themonth" might not be applicable to modern society due to improved quality of life, the value of "sitting-the-month" should not be dismissed or overlooked Liu, et al. [13]. Since most Chinese women are raised with the belief of "sitting-the-month" and maintaining warmth, failure to do so might increase maternal stress and family discord Liu, et al. [13]. In addition, "sitting-the-month" is a valuable time for the new mom to be with her newborn, to be honored and to receive emotional support from her extended family Chen [22]. The implication for nursing practice is to ask patients and families more open-ended questions in order to gain further information about their beliefs, values, assumptions, and expectations. It is not appropriate to judge or criticize patient's customs even though they might seem "silly" to healthcare professionals. It can be concluded that people from mainland China and Australia hold different health values, beliefs and practice due to cultural differences [2527]. The general health literacy in China is much lower than that of Australia. These two countries have different social opinions regarding parents staying in a nursing home. In addition, cultural specific values also affect people's expectation towards healthcare system and healthcare services [28-32]. They further shape unique patient-doctor/nurse relationships. All in all, to provide cultural sensitive nursing care, Registered nurses in Australia need to be aware of cultural clashes and take nursing strategies to address cross-cultural issues accordingly.

\section{References}

1. (2015) Australian Nursing \& Midwifery Federation. Nurses speak out about violence in the workplace.

2. Chung VCH, Ma HX, Lau CH, Wong SYS, Yeoh EK, et al. (2014) Views on traditional Chinese medicine amongst Chinese population: A systematic review of qualitative and quantitative studies. Health Expectations 17(5): 622 .

3. Zhang F, Jiang Z, Zhang W (2016) Tea therapy rescue the lung injury from atmospheric particulate matters. The Gerontologist: 56(3): 134 .
4. Chang JS, Wang KC, Yeh CF, Shieh DE, Chiang LC (2013) Fresh ginger (zingiber officinale) has anti-viral activity against human respiratory syncytial virus in human respiratory tract cell lines. Journal of Ethnopharmacology 145(1): 146-151.

5. Zhao L, Zhang XY, Bai GY, Wang, YG (2014) Violence against doctors in China. The Lancet 384 (9945): 744.

6. E (2012) Understanding the Australian health care system ( $\left.2^{\text {nd }} e d n.\right)$. Sydney, Chatswood, NSW: Churchill Livingstone.

7. Hao R, Zhao R, Qiu S, Wang L, Song H (2015) Antibiotics crisis in China. Science 348(6239): 1100-1101.

8. Capon A, Smith W \& Gillespie JA (2012) Navigating public health chemicals policy in Australia: A policy maker's and practitioner's guide. NSW Public Health Bulletin 23(12): 217-227.

9. Hodge A (2018) Professional transitions in nursing: A guide to practice in the Australian healthcare system. New South Wales, NSW: Allen \& Unwin.

10. Wu Y, Wang L, Cai Z, Bao L, Ai P, et al. (2017) Prevalence and risk factors of low health literacy: A community-based study in shanghai, China. International Journal of Environmental Research and Public Health, 14(6): 55-59.

11. Zhang D, Wu S, Zhang Y, Yang P, MacIntyre CR, Seale H, Wang Q (2015) Health literacy in beijing: An assessment of adults' knowledge and skills regarding communicable diseases. BMC Public Health 15(1): 8-12.

12. Wang X, Guo, H, Wang L, Li X, Huang M, et al. (2015) Investigation of residents' health literacy status and its risk factors in Jiangsu province of China. Asia-Pacific Journal of Public Health 27(2): 2764-2772.

13. Li X, Ning N, Hao Y, Sun H, Gao L, et al. (2013) Health literacy in rural areas of China: Hypertension knowledge survey. International Journal of Environmental Research and Public Health 10(3): 1125.

14. Gao YL, Wei YB, Shen YD, Tang YY \& Yang JR (2014) China's empty nest elderly need better care. Journal of the American Geriatrics Society 62(9): 1821-1822.

15. Chen S (2011) Zuoyuezi sitting the month in Taiwan: Implications for intergenerational relations (Doctoral dissertation). Retrieved from ProQuest Dissertations and Theses. (UMI 3463404).

16. Smith CD, Fisher C, Mercer A (2011) Rediscovering nursing: A study of overseas nurses working in Western Australia. Nursing \& Health Science 13(3): 289-295.

17. Jing W, Otten H, Sullivan L, Lovell Simons L, Granek Catarivas M, et al. (2013) Improving the doctor-patient relationship in China: The role of balint groups. The International Journal of Psychiatry in Medicine, 46(4): 417-427.

18. The Economist (2012) Heartless attacks violence against doctors. The Economist 404 (8794): 38.

19. Cashmore AW, Indig D, Hampton SE, Hegney DG, Jalaludin BB (2012) Workplace violence in a large correctional health service in New South Wales, Australia: A retrospective review of incident management records. BMC Health Services Research 12: 245.

20.(2018) China Development Research Foundation (Ed). Reforming China's healthcare system (1 $1^{\text {st }}$ Edn.). New York, NY: Routledge.

21. Ku TK, Maude PM, Welch A (2011) FP05-2 culture and stigma of mental illness: An analysis of Chinese Australian community. Asian Journal of Psychiatry 4(1): 34.

22. Chen S (2011) Zuoyuezi sitting the month in Taiwan: Implications for intergenerational relations (Doctoral dissertation). Retrieved from ProQuest Dissertations and Theses. (UMI 3463404).

23. Liu YQ Petrini M, Maloni JA (2015) Doing the month: postpartum practices in Chinese women. Nursing \& Health Sciences 17(5): 5-14.

24. Yan Y (2018) The ethics and politics of patient-physician mistrust in contemporary China. Developing World Bioethics 18(1): 7-15. 
25. Lin N (2015) The restorative role of apology in resolving medical disputes: Lessons from Chinese legal culture. Journal of Bioethical Inquiry 12(4): 699.

26. Nickell J (2012) Traditional Chinese medicine: views east and west Skeptical Inquirer 36(5): 18.

27. (2018) Nursing and Midwifery Board of Australia (NMBA), Code of ethics for nurses.

28. Song Y (2014) Losing an only child: The one-child policy and elderly care in China. Reproductive Health Matters 22(43): 113-124.

\section{ISSN: 2574-1241}

DOI: $10.26717 / B J S T R .2018 .09 .001856$

Jie Li Li. Biomed J Sci \& Tech Res

(c) 9 This work is licensed under Creative

Submission Link: https://biomedres.us/submit-manuscript.php
29. Tan ES (2011) FP05-1 the weeping Chinese woman syndrome. Asian Journal of Psychiatry 4(1): 34.

30. Dong X (2017) Perspectives on care for the elderly in China-reply. JAMA Internal Medicine 177(3): 444-445.

31. (2018) Australian Institute of Health and Welfare. Australia's health.

32. (2018) Australian Institute of Health and Welfar. Immunization rates for vaccines in the national schedule (children).



